\title{
GAME THEORY \\ IN ECONOMICS EDUCATION
}

\author{
Elena Yevsyeyeva, Olena Skafa
}

\begin{abstract}
The article is devoted to the application of skills gained by students in Mathematics for Business and Economics to solve problems of the game theory. An application of the entry game analysis is considered. A real-life problem is described which consists of the application of the zero-sum game to an auction situation, followed by finding the optimal solution with respective recommendations.
\end{abstract}

Keywords: game theory, zero-sum game, economics education, mathematics for business and economics.

JEL Classification: C71.

DOI: $10.15611 / \mathrm{dm} .2016 .13 .06$.

\section{Introduction}

At the present time, higher education in Economics should be tailored to the current needs of the labor market, with which a student will be confronted after university graduation. All disciplines in the curriculum of Master's program contain a variety of professionally related mathematical problems, which acquaint students with the subtleties of their future career. The course "Mathematics of Economics and Business" falls under the description mentioned above. It embraces such topics as differential and integral calculus of one and several variables functions, linear and vector algebra, differential equations, linear programming and vector spaces [Werner, Sotskov 2006]. Yet there is no game theory in the curriculum of this course, whilst at the same time it is an important tool in economics. The application of the Game Theory may be very useful in preparing a Master's thesis in economics.

In this paper the game theoretic model will be described and applied to a real-life situation. To begin with, the basic concepts of a game theory will be briefly discussed with the classification of the games. Afterwards, the

Elena Yevsyeyeva, Olena Skafa

The Higher Mathematics and Methods of Mathematics Teaching Department, Donetsk National University

donntu@gmail.com 
general case of a two-person zero-sum game will be considered with the further derivation of pure and mixed strategies Nash Equilibrium with the geometrical interpretation of the $2 \times 2$ game. We have taken the two-person zero-sum game as it wholly represents the current situation on the market and gives the possibility to create a robust and accurate theoretical model. Finally, the last step is to apply of the two-person zero-sum game to a real life situation and to give recommendations according to the optimal strategy.

\section{Game Theory in the curriculum of mathematics}

One of the tools to optimize the process of decision making is game theory, which in the modern economy has a variety of practical applications. However, the analysis of Masters of Economics training curriculum showed a lack of subjects that would give students the ability to make optimization decisions under conditions of uncertainty. Higher mathematics, probability theory and mathematical statistics are intended to equip students with the mathematical apparatus for constructing deterministic, stochastic and statistical models of economic processes and phenomena, including the solution of optimization problems. In the solution of management tasks in cases when it is impossible to find the exact optimal solution of the optimization methods, including methods of game theory, represent an auxiliary tool which allows to evaluate each of the possible variants of development of events, and therefore make an informed decision - which is especially important for future managers.

Tasks of game theory relate to decision-making problems under uncertainty and risk. The uncertainty of the game results is due to several factors. First, as a rule, the number of possible scenarios is very large, therefore it is impossible to predict the outcome of the game. Secondly, a significant impact on the progress and results of games comes from random factors which cannot be predicted. Third, a source of uncertainty is the lack of information about the actions of the enemy. In addition, this uncertainty to a certain extent may also relate to the goal, which seeks to achieve the subject of the activity. It is not always a goal that can be expressed unambiguously, and even more so by one measure. It is clear that when the initial conditions of the problem contain a large number of uncertain parameters, then mathematical research cannot give clear justification rational decisions, but in the absence of full certainty, a quantitative analysis gives the scientific basis for decisionmaking. 
Elements of game theory are also studied by students in such courses as "Mathematical programming", "Operations Research", "Economicmathematical Methods and Models", etc. However, graduates usually have a very superficial understanding of the subject and methods of game theory - even if they studied this discipline in the curriculum - due to the fact that the mathematical apparatus of game theory seems to them difficult, and examples of its application divorced from practice.

\subsection{Basic concepts of Game Theory}

The Game Theory was first systematically expounded by Neumann and Morgenstern and published in 1944 in the book "Theory of Games and Economic Behaviour”, although some results had been published 20 years earlier. Neumann and Morgenstern had written very original book which contained mainly economic examples, as economic problems could be more easily described by the means of numbers. During and after the Second World War, the military were seriously interested in game theory. They had seen its mathematical tools for the study of strategic problems and a tool for decision making. Now the scope of game theory has significantly expanded. Thus, game theory is used in social sciences, in psychology, to analyze trade agreements and negotiations, to study the principles of forming coalitions and more [Dixit, Skeath 2004].

It is known that any economic system does not function in isolation and enters into some economic relationships with other entities at some point of time. This part of the paper describes the situations with multiple participants, when the objective value function for each participant depends not only on his/her own behavior, but also on the actions of other actors.

In a market relationship, individuals likely to have conflict situations where two or more individuals (groups) have contrary goals and interests, and the result of each of them depends on the actions of the other. A classic example of the conflict situation is the seller - buyer relationship. More complex situations arise when associations or coalitions are involved in the dispute. Note that the participants should have opposing goals in a game situation. For example, two companies that provide similar services can be combined to face opponents jointly [Dixit, Skeath 2004].

Game theory is a mathematical tool that examines conflicts and the situations of the joint actions of several participants. The main task of game theory is to develop recommendations regarding rational behavior of the players. 
The real conflict situations are quite complex and burdened with a large number of minor factors which complicate the analysis. That is why simplified models of conflict situations are made which are called - games. A characteristic feature of the mathematical model is the existence of a game situation. Firstly, the game should consist of several participants, called players; secondly, the description of the possible actions of each party, which are called strategies; and thirdly, the defined results of actions for each player which can be described by the functions. The goal is to find the optimal strategy that provides the highest possible average payoff in cases of the multiple repetition of the game [Varian Hal 2014].

\subsection{Classification of games}

The classification of games is conducted by the selected criterion. Games can vary depending on the number of players, number of strategies, the properties of the payoff functions, and the possibilities of interaction between players.

A game could be a one-person, two-person, or $n$-person (with $n$ greater than two) game. Depending on the number of strategies, finite and nonfinite games are distinguished. If each player has a finite number of strategies, the game is finite, otherwise - infinite. If the gain of one player is the loss of another, we have a zero-sum game. These games are characterized by anti-depending interests of the parties and a conflict situation. In cases of a possible combination of interests and players agreement on the choice between them, one can talk about strategies for a cooperative game. When players are not able or not willing to coordinate their actions, the game is called non-cooperative [Varian Hal 2014].

The most frequent case in game theory is with the 2-player games, in which the gain of one party is the loss of the other, or as was described earlier, a zero-sum game. This situation is typical in management, marketing, and advertising services, where workers make decisions under conditions of intense competition and incomplete information every day. The main goal of solving such problems is to develop recommendations regarding the selection of the optimal strategies of the parties on the basis of the methodological approaches of the game theory [Hazewinkel 2001]. 


\subsection{General case}

Assume we have two players A and B (two-person zero-sum game). Each player chooses one of the possible strategies: denote Player's A strategies by $A_{i}(i=\overline{i, m})$, and the Player's B strategies by $B_{j}(j=\overline{1, n})$.

Results (payoffs) for all possible variations of the game are given by special functions that depend on the strategies of other players usually in the form of a payoff matrix.

Let $\varphi_{1}\left(A_{i} ; B_{j}\right)(i=\overline{i, m} ; j=\overline{i, n})-$ is when Player A wins; and $\varphi_{2}\left(A_{i} ; B_{j}\right)$ $(i=\overline{i, m} ; j=\overline{i, n})-$ is when Player B wins.

Since we have a zero-sum game $\varphi_{1}\left(A_{i} ; B_{j}\right)+\varphi_{2}\left(A_{i} ; B_{j}\right) \equiv 0$. We have $\varphi_{1}\left(A_{i} ; B_{j}\right)=\varphi\left(A_{i} ; B_{j}\right)$, and $\varphi_{2}\left(A_{i} ; B_{j}\right)=-\varphi\left(A_{i} ; B_{j}\right)$. Thus, the goal of Player $\mathrm{A}$ is to maximize the value $\varphi\left(A_{i} ; B_{j}\right)$ and Player $\mathrm{B}-$ minimize it. Suppose that $\varphi\left(A_{i} ; B_{j}\right)=a_{i j}$, we have the matrix A:

$$
A=\left(\begin{array}{cccc}
a_{11} & a_{12} & \ldots & a_{1 n} \\
a_{21} & a_{22} & \ldots & a_{2 n} \\
\ldots & \ldots & \ldots & \ldots \\
a_{m 1} & a_{m 2} & \ldots & a_{m n}
\end{array}\right),
$$

where rows correspond to the strategies $A_{i}$ and columns to the strategies $B_{j}$. The matrix $\mathrm{A}$ is called the payoff matrix. An element of this matrix $a_{i j}$ is when Player A wins if he/she had chosen $A_{i}$ strategy, and Player B had chosen the $B_{j}$ strategy [Savin, Nakonechnaja 2003].

From the many game theory criteria for selecting rational solutions, the most common is the pessimistic criterion of Minimax-Maximin. The essence of this criterion is as follows:

Let Player A chose strategy $A_{i}$, then in the worst case it will receive a payoff equal to min $a_{i j}$, i.e. even if Player B would have known the strategy of Player A. Anticipating this possibility, Player A has to choose the strategy to maximize his/her minimum payoffs, such as:

$$
a=\max _{i} \min _{j} a_{i j}
$$

Such a strategy of Player $\mathrm{A}$ is denoted by $A_{i_{0}}$ and is called maximin, and the value of the guaranteed payoff of a player is called the lower value of the game. Player B who loses the payoffs in the amount of the elements in the matrix, conversely should choose a strategy that minimizes its maxi- 
mum possible loss of all options to Player A. Player B's strategy is denoted by $B_{j o}$ and is called the minimax. The amount of the loss is called the upper value of the game:

$$
\beta=\min _{j} \max _{i} a_{i j}
$$

The optimal solution to this problem is achieved when there is no incentive to change the chosen strategy for both parties, as its opponent can choose a different response strategy that will ensure a better outcome [Hazewinkel 2001].

If $\max _{i} \min _{j} a_{i j}=\min _{j} \max _{i} a_{i j}=v$, or in other words $a=\beta=v$, the game is called well-defined. In this case Player A winning (Player B losing) is an element of the matrix. Such games are called games with saddle points, and the element whose value is equal to Player A winning (Player B losing) is a saddle point (Nash Equilibrium). In this situation, the optimal solution for the game for both sides is the choice of only one of the possible pure strategies - maximin for Player A and minimax for Player B, that is if one of the players follows the optimal strategy, for the second player the deviation from its optimal strategy cannot be beneficial [Hazewinkel 2001].

Minimax and maximin strategies are called pessimistic strategies. The choice of the optimal strategy for each player is based on the assumption that he/she will act under the worst possible conditions. Clearly, in this case, the choice of such a strategy may not satisfy the players. Let Player A choose the maximin strategy, assume also that Player B knows the opponent's strategy choice, then it is expedient to choose the strategy that will increase his/her payoffs. In turn, Player A is also aware of the change in strategy of Player B and chooses the other strategy which will give him/her the opportunity to benefit to a higher amount than Player B, etc. The possibility of such a development occurs because the minimax and maximin strategies are not stable. That is, the circumstances in which both players use the minimax or maximin strategies are not beneficial for both in the case when one of the players changes the optimal strategy [Szép, Forgó 1985].

However, this instability is not inherent to all games with saddle points. In some cases, the saddle point corresponds to a stable maximin and minimax strategy. In this case, the deviation from the optimal strategy of one player causes changes in the won amount, which is disadvantageous for this player as his/her state either stays unchanged or worsens. Thus in general it cannot be said that games with a saddle point can determine the optimal sustainable strategy [Savin, Nakonechnaja 2003]. 


\subsection{Mixed strategies}

Finite games usually do not have a saddle point. If the game has no saddle point, i.e. $\alpha \neq \beta$ and $\alpha \leq v \leq \beta$, its maximin - minimax strategies are not optimal. This means that each party can improve its results by choosing a different approach. The optimal solution for such a game is mixed strategies, which are the specific combinations of the original "pure" strategies. That is, a mixed strategy involves the use of several "pure" strategies with varying frequencies. The probabilities (frequencies) of each strategy are given with vectors [Savin, Nakonechnaja 2003]:

for Player A - vector $X=\left(x_{1}, x_{2}, \ldots x_{m}\right)$, where $\sum_{i=1}^{m} x_{i}=1$,

for Player B - vector $Y=\left(y_{1}, y_{2}, \ldots y_{n}\right)$, where $\sum_{j=1}^{n} y_{j}=1, x_{i} \geq 0(i=\overline{1, m})$; $y_{j} \geq 0(j=\overline{1, n})$.

It appears that when the mixed strategies are used, for every finite game one can find a couple of stable optimal strategies. The main theorem of game theory determines the existence of a solution. The main theorem of game theory says that every finite game has at least one solution which is possible in mixed strategies.

Suppose we have a finite matrix game with the payoff matrix (1). According to the theorem, optimal mixed strategies for players are determined by vectors $X^{*}=\left(x_{1}^{*}, x_{2}^{*}, \ldots, x_{m}^{*}\right)$ and $Y^{*}=\left(y_{1}^{*}, y_{2}^{*}, \ldots, y_{n}^{*}\right)$, which give the possibility to receive the win: $\alpha \leq v \leq \beta$.

Using the optimal mixed strategy should provide Player A with a certain payoff that is not less than the value of the game. Mathematically, this condition is written as:

$$
\sum_{i=1}^{m} a_{i j} x_{i}^{*} \geq v \quad(j=\overline{1, n}) .
$$

On the other hand, the use of an optimal mixed strategy for Player B should provide, for any strategy that Player A chooses, the loss that is not exceeding the value of the game, that is:

$$
\sum_{j=1}^{n} a_{i j} y_{j}^{*} \leq v(i=\overline{1, m}) .
$$

These relationships are used to find the solution of the game. In the case of mixed strategies the calculated optimal strategy is always stable, i.e. if 
one player follows his/her optimal mixed strategy, its payoff remains constant and equal to the value of the game $v$, no matter which of the possible mixed strategies are chosen by another player. The simplest case of a finite game is a $2 \times 2$ game where each participant has two strategies.

Consider the case when the game has no saddle point, i.e. $\alpha \neq \beta$. We need to find the mixed strategies and the value of the game. Denote the desired probabilities value for the Player A in the "pure" strategies with $X^{*}=\left(x_{1}^{*}, x_{2}^{*}\right)$ and for Player B with $Y^{*}=\left(y_{1}^{*}, y_{2}^{*}\right)$.

According to the main game theory theorem, if the player sticks to its optimal strategy, the payoff will be equal to the value of the game. So if Player A sticks to his/her optimal strategy $X^{*}=\left(x_{1}^{*}, x_{2}^{*}\right)$, then:

$$
\left\{\begin{array}{l}
a_{11} x_{1}^{*}+a_{21} x_{2}^{*}=v \\
a_{12} x_{1}^{*}+a_{22} x_{2}^{*}=v
\end{array}\right.
$$

Since $x_{1}^{*}+x_{2}^{*}=1$, then $x_{2}^{*}=1-x_{1}^{*}$. Substituting this expression into the system (6), we obtain:

$$
\left\{\begin{array}{l}
a_{11} x_{1}^{*}+a_{21}\left(1-x_{1}^{*}\right)=v ; \\
a_{12} x_{1}^{*}+a_{22}\left(1-x_{1}^{*}\right)=v .
\end{array} \Rightarrow a_{11} x_{1}^{*}+a_{21}\left(1-x_{1}^{*}\right)=a_{12} x_{1}^{*}+a_{22}\left(1-x_{1}^{*}\right) .\right.
$$

Solving this equation for $x_{1}^{*}$, we have:

$$
x_{1}^{*}=\frac{a_{22}-a_{21}}{a_{11}+a_{22}-a_{12}-a_{21}},
$$

then:

$$
x_{2}^{*}=1-x_{1}^{*}=\frac{a_{11}-a_{12}}{a_{11}+a_{22}-a_{12}-a_{21}} .
$$

Having made the same calculations for Player $\mathrm{B}$, we obtain:

$$
\left\{\begin{array}{l}
a_{11} y_{1}^{*}+a_{12} y_{2}^{*}=v \\
a_{21} y_{1}^{*}+a_{22} y_{2}^{*}=v
\end{array}\right.
$$

As $y_{1}^{*}+y_{2}^{*}=1$, then $y_{2}^{*}=1-y_{1}^{*}$

$$
\left\{\begin{array}{l}
a_{11} y_{1}^{*}+a_{12}\left(1-y_{1}^{*}\right)=v ; \\
a_{21} y_{1}^{*}+a_{22}\left(1-y_{1}^{*}\right)=v .
\end{array} \Rightarrow a_{11} y_{1}^{*}+a_{12}\left(1-y_{1}^{*}\right)=a_{21} y_{1}^{*}+a_{22}\left(1-y_{1}^{*}\right)\right.
$$


Solving this equation for $y_{1}^{*}$, we have:

$$
y_{1}^{*}=\frac{a_{22}-a_{12}}{a_{11}+a_{22}-a_{12}-a_{21}},
$$

then:

$$
y_{2}^{*}=1-\frac{a_{22}-a_{12}}{a_{11}+a_{22}-a_{12}-a_{21}}=\frac{a_{11}-a_{21}}{a_{11}+a_{22}-a_{12}-a_{21}} .
$$

The value of the game $v$ could be found by plugging the values $x_{1}^{*}, x_{2}^{*}$ (or $y_{1}^{*}, y_{2}^{*}$ ) to any of the equations (6) or (9):

$$
v=\frac{a_{22} a_{11}-a_{12} a_{21}}{a_{11}+a_{22}-a_{12}-a_{21}} .
$$

\section{Application of the model}

The next step is the application of the above model to the real situation. We will derive pure and mixed strategies Nash Equilibrium with the corresponding geometric representation, and finally, the recommendations according to the optimal strategy will be made. To find the optimal strategy for the company, the first step would be to build the payoff matrix (1). In this part of the paper we consider the zero-sum game - a game in which one player's gains result only from another player's equivalent losses. We will consider this model from the point of view of the company MWE.

Let us assume that some Ukrainian metallurgical company invests in two new projects and the company is organizing a tender to find the contractors for the engineering and construction.

The first project $\left(A_{1}\right)$ is the engineering and erection of a new rolling mill in the central region of Ukraine. The second project $\left(A_{2}\right)$ is an upgrade and adjustment of the existing rolling mill in the eastern region of Ukraine. The first project is more expensive and, of course, is more valuable to the companies participating in the tender.

Three engineering companies which are represented in this segment and interested in the Ukrainian market are participating in the tender. Let us assume that these companies are: MWE, Siemens and SMS Meer. Siemens is the clear favorite in getting a larger project in the Ukrainian market as its market share is much larger than that of MWE or SMS Meer.

In the proposed model we consider two possible scenarios: when Siemens and MWE enter into a strategic interaction and are competing for the 
projects - scenario $\left(B_{1}\right)$; and the scenario $\left(B_{2}\right)$, when now SMS Meer and MWE are competing for the projects.

In the payoff matrix (1), $A_{1}$ and $A_{2}$ are projects 1 and 2 (Table 1). The expected return which the companies could get from project $A_{1}$ is 100000 EUR, the expected return for the second project $A_{2}$ is approximately 70000 EUR. Strategy $B_{1}$ is a situation in which MWE and Siemens are vying on the market, while strategy $B_{2}$ is a situation in which MWE and SMS Meer are competing for the projects.

First, let us consider situation $B_{1}$. In situation $A_{1} B_{1}$, when there are Siemens and MWE on the market, the probability of obtaining an order for constructing a plant for Siemens is much higher than that of MWE. This is due to the fact that Siemens is better known and it has extensive experience in the construction of plants from scratch. The probability of receiving an order for Siemens will be $80 \%$ and $20 \%$ for MWE. For the $A_{2} B_{1}$ situation, MWE has a slightly higher chance of getting this project because MWE has significant experience in reconstructing the factories and setting up equipment and has firmly established itself in this area. In this case the probability of receiving an order for Siemens will be 55\% and for MWE 45\%.

This means that in the cell $A_{1} B_{1}$ in the payoff matrix (1) we write:

$$
100000 E U R *(0.2)=20000 E U R \text {. }
$$

And in the cell $A_{2} B_{1}: 70000 E U R *(0.45)=31500$ EUR.

The values in cells are the expected payoffs for MWE and the expected losses for Siemens at the same time.

Now let us consider situation $B_{2}$. In this situation MWE and SMS Meer are vying in the market. These two firms are relatively new to the Ukrainian market, and therefore the probability of receiving an order for constructing a new plant for both firms will be the same, at 50\%. However, in the situation $A_{2} B_{2}$, SMS Meer has a slight advantage as it has more experience in adapting in the Eastern region of Ukraine (a plant in Donetsk), so the probability of receiving an order for MWE is $40 \%$ and for SMS Meer $-60 \%$.

Table 1. The playoff matrix of the game

\begin{tabular}{|c|c|c|c|}
\hline \multirow{2}{*}{$i$} & $A_{i}$ & \multicolumn{2}{|c|}{$B_{j}$} \\
\cline { 3 - 4 } & & $B_{1}$ (MWE/Siemens) & $B_{2}$ (MWE/SMS Meer) \\
\hline 1 & $A_{1}(100000$ EUR) & 0.2658 & 0.0748 \\
\hline 2 & $A_{2}(70000$ EUR) & 0.4986 & 0.0636 \\
\hline \multicolumn{2}{|c}{${ }^{*} A_{i}$ - projects; $B_{j}$ - scenario. }
\end{tabular}

Source: own elaborations. 
This means that in the cell $A_{1} B_{2}$ in the payoff matrix (1) we write: $100000 E U R \times(0.5)=50000 E U R$, and in the cell $A_{2} B_{2}$ :

70000 EUR $\times(0.4)=28000 E U R$.

Thus finally in Table 1 we obtain the following payoff matrix (1).

Make sure that the game does not have a saddle point taking into account (2)-(3): $\max \{\min (20000 ; 50000) ; \min (31500 ; 28000)\}=\max \{20000 ; 28000\}=28000=\alpha$, $\min \{\max (20000 ; 31500) ; \max (50000 ; 28000)\}=\min \{31500 ; 50000\}=315000=\beta$.

If $\alpha \neq \beta$, this means that the game does not have a saddle point. Taking into account (7)-(11) we obtain:

$$
\begin{aligned}
x_{1}^{*}=\frac{a_{22}-a_{21}}{a_{11}+a_{22}-a_{12}-a_{21}}=\frac{28000-31500}{20000+28000-50000-31500}=0.104 ; \\
x_{1}^{*}=\frac{a_{22}-a_{12}}{a_{11}+a_{22}-a_{12}-a_{21}}=\frac{28000-50000}{20000+28000-50000-31500}=0.65 ; \\
y_{2}^{*}=1-0.65=0.35 .
\end{aligned}
$$

The value of the game (12) is:

$$
v=\frac{a_{22} a_{11}-a_{12} a_{21}}{a_{11}+a_{22}-a_{12}-a_{21}}=\frac{28000 \cdot 20000-50000 \cdot 31500}{20000+28000-50000-31500}=30298.5 .
$$

The solution of the game means that MWE should mix its pure strategies, which consist of choosing the $A_{1}$ project jointly with Siemens with the probability 0.104 and the $A_{2}$ project jointly with Siemens with the probability 0.896. Project $A_{1}$ together with SMS Meer, MWE should choose with probability 0.65 and project $A_{2}$ with SMS Meer, MWE chooses with the probability 0.35 . Under these conditions, the expected gain will be equal to the value of the game which is 30298.5 Euro. This means that if MWE has to choose which strategy to follow, it would give preference to the strategies with high probabilities. That is, $A_{2}$ together with Siemens, and $A_{1}$ together with SMS Meer. These results are summarized in Table 2.

Note on the $x$-axis the segment with the length equal to one (Figure 1). The left part of the segment corresponds to strategy $A_{1}$ and the right end corresponds to strategy $A_{2}$. All the intermediate points of the segment correspond to the mixed strategies of MWE. The probability $x_{1}=0.104$ of strategy $A_{1}$ will be equal to the distance from point $P$ to the right end of the segment, and the probability $x_{2}=0.896$ of $A_{2}$ strategy is the distance to the left end of the segment. Let us draw two perpendiculars to the $x$-axis through the 
points $A_{1}$ and $A_{2}$ - axis I and axis II. The first will correspond to the gains that a firm could get choosing strategy $A_{1}$ and the second - to strategy $A_{2}$.

Table 2. Mixed strategy distribution for MWE

\begin{tabular}{|c|c|c|c|}
\hline \multirow{2}{*}{$i$} & \multirow{2}{*}{ Competing firm } & \multicolumn{2}{|c|}{ Projects } \\
\cline { 3 - 4 } & & $A_{1}$ & $A_{2}$ \\
\hline 1 & Siemens & $x_{1}^{*}=0.104$ & $x_{2}^{*}=0.896$ \\
\hline 2 & SMS Meer & $y_{1}^{*}=0.65$ & $y_{2}^{*}=0.35$ \\
\hline
\end{tabular}

${ }^{*} X^{*}=\left(x_{1}^{*}, x_{2}^{*}\right)-$ optimal strategy for Siemens; $Y^{*}=\left(y_{1}^{*}, y_{2}^{*}\right)-$ optimal strategy for SMS Meer.

Source: own elaborations.

One can also give a clear geometric interpretation for the solution of the $2 \times 2$ game. Following the instructions above one can get Figure 1 :

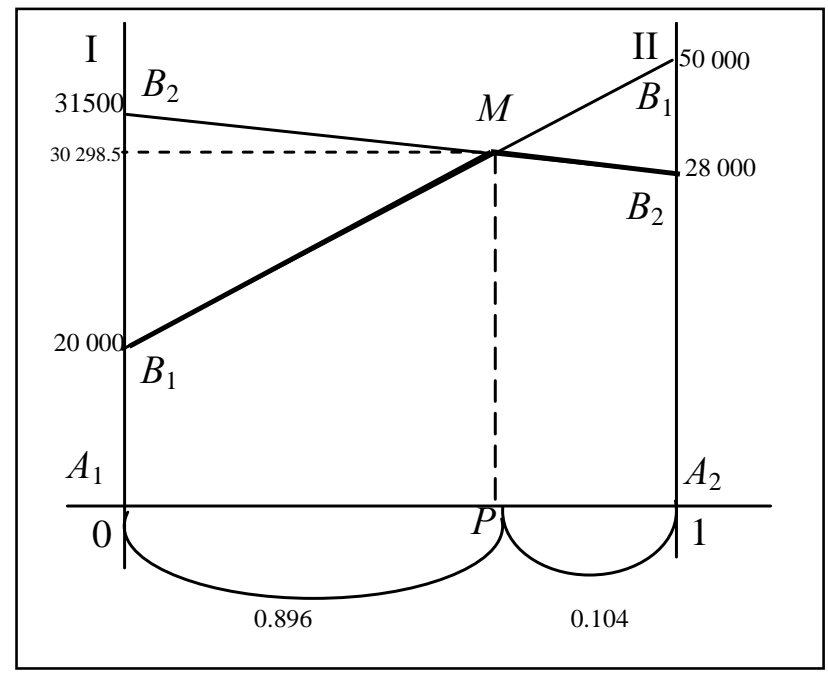

Fig. 1. Geometric interpretation of the game

Source: own elaborations.

Let us draw the lines which correspond to strategy $B_{1}$. The length of the segment $A_{1} B_{1}$ equals $a_{11}=20000$, while the length of the segment $A_{2} B_{1}$ equals $a_{12}=50$ 000. Similarly, draw the lines which correspond to strategy $B_{2}$.

We need to find the optimal strategy $X^{*}$, for which the minimum payoff of MWE will be maximized. To do this, let us draw a bold line (Figure 1) which corresponds to the lower bound of winning if strategies 
$B_{1}$ and $B_{2}$ are selected, i.e. the kinked line $B_{1} M B_{2}$. This line includes all the minimum gains of MWE at any of its mixed strategies. It is obvious that the best possible minimum value in this example is at point $M$, and generally corresponds to the point where the curve that indicates the minimum payoff of MWE gets its maximum value. The ordinate of this point is the value of the game and equals $v=30298.5$. The distance from the left end of the segment $x_{2}$ and the distance to the right end of the segment $x_{1}$ respectively, are equal to the probabilities of strategies $A_{2}$ and $A_{1}$.

As a result, it should be said that after considering the theoretic entry situation we came to the conclusion that for MWE the optimal solution would be to mix its pure strategies, i.e. while competing with Siemens, the choose $A_{1}$ project with the probability 0.104 and the $\mathrm{A}_{2}$ project with the probability 0.896. Project $A_{1}$ together with SMS Meer, MWE should choose with probability 0.65 and project $A_{2}$ with SMS Meer, MWE chooses with the probability 0.35 . Under these conditions, the expected gain will be equal to the value of the game which is 30298.5 Euro. This means that if MWE has to choose which strategy to follow, it will give preference to the strategies with high probabilities, that is $A_{2}$ together with Siemens, and $A_{1}$ together with SMS Meer.

\section{Conclusion}

Thus, we have shown that game theory is very important in economic education and can be used for the solution of optimization problems. At the same time, the fact that game theory is not included in the traditional course of "Mathematics of Economics and Business" complicates its use.

Hermann Hesse said: “...Game is not a philosophy and not a religion, it is a special discipline, which is very close to art by its nature..." [Hesse, reprinted 2002]. Students are required to have versatile skills and a creative approach to self-learn and apply new methods of the game theories.

Ways to improve education at the tertiary level might be to develop learning technologies based on the activity based approach [Yevsyeyeva 2012, 2013] and to design heuristic problems systems [Skafa 2015] that help to develop the creative abilities of students.

The methodology for the formation of skills of game theory among students of economics in situations where the educational program does not provide for the study of this theory within the framework of a separate discipline can be expressed by the following provisions: 
1. The goals of teaching game theory are based on the state standard and should be recognized by students. Therefore, one of the important tasks for creating a goal-based theory of games in teaching mathematical disciplines is the use of professionally-directed problems that appeal to the concept game of game theory.

2. The content of training is determined by the game-theoretic models used in the professional work of economists and managers.

3. Training methods should include productive search methods such as the orientation method, heuristic methods, the mathematical modeling method, and the method of highlighting the learning activities performed at the main stages of the learning activity to solve professionally-directed problems.

4. The priority organizational forms, in which it is possible to form students' ability to make decisions in conditions of risk and uncertainty, is the independent and scientific research work of students.

5. The teaching aids should be supplemented by a distance course or the teaching aid "Theory of games in the professional training of a manager and an economist”, developed on the basis of an active learning technology.

\section{Bibliography}

Dixit A., Skeath S. (2004). Games of strategy. W. Norton \& Company.

Evseeva E.(2015). Game theory teaching methods for students specializing in economics and management. Didactics of mathematics: Problems and Investigations. No. 43. Pp. 47-55.

Hazewinkel M. (2001). Theory of Games. [In:] Encyclopedia of Mathematics. Springer.

Hesse H. (reprinted 2002). Glass Bead Game. Holt Paperbacks. Reprint edition.

Savin S., Nakonechnaja S. (2003). Mathematical programming. Kyiv. KNEU.

Skafa O. (2015). Heuristic construction as a management learning tool in mathematics.

Didactics of mathematics: Problems and Investigations. No. 43. Pp. 21-26.

Szép J., Forgó F. (1985). Introduction to the Theory of Games. Reidel.

Varian Hal R. (2014). Intermediate Microeconomics: A Modern Approach. Eighth Edition. W. Norton \& Company.

Werner F., Sotskov Y. (2006). Mathematics of Economics and Business. Taylor \& Francis e-Library.

Yevsyeyeva E. (2012). Planning methodical system of teaching mathematics at technical university on the basis of activity approach. Didactics of Mathematics: Problems and Investigations. No. 37. Pp. 7-16.

Yevsyeyeva E. (2013). The conception of the mathematics teaching planning and organization of the higher technical educational school's students on the principles of activities approach. Didactics of mathematics: Problems and Investigations. No. 40. Pp. 108-117. 